\title{
Brigide Schwarz
}

\section{Dispense der Kanzlei Eugens IV. (1431-1447)}

Mein Thema sind Dispense vom Geburtsmakel in den „Registern der Kanzlei“ (zum Begriff später) unter Eugen IV. ${ }^{1}$ Nachdem jetzt die Register der Pönitentiarie zugänglich geworden sind, über die uns Monsignore Tamburini berichtet hat, kann man an eine vergleichende Betrachtung der Dispensations-Praxis der beiden kurialen „Behörden“2 herangehen, die bisher anhand der relativ wenigen zugänglichen Original-Ausfertigungen der Pönitentiarie ${ }^{3}$ nicht möglich war.

Kanzlei ${ }^{4}$ und Pönitentiarie erteilten bzw. fertigten die weitaus meisten ,apostolischen“ Dispense aus. Ehe ich näher darauf eingehe, kurz zu den konkurrierenden Instanzen: (1) Zunächst war nach gemeinem Recht der Ordinarius zuständig für Dispensierung illegitim geborener Ordinanden, aber mit der Einschränkung ad minores ordines und ad unum beneficium simplex. ${ }^{5}$. Das war zuwenig für Kleriker mit Ehrgeiz. Hier ist daran zu erinnern, daß fast alle, die um eine apostolische Dispens einkamen, dies deswegen taten, weil sie Kleriker werden wollten bzw. als Kleriker nach höheren Weihen und/oder Benefizien strebten. Davon waren wieder die weitaus meisten Säkularkleriker, eine Minderzahl Regu-

1 Um Anmerkungen vermehrte, geringfügig veränderte Fassung des Vortrags vom 7. April 1992. Die Dispense in den Kanzleiregistern dieses Pontifikats, soweit für „deutsche“ Petenten erteilt, sind mir aus der Bearbeitung des Repertorium Germanicum dieses Papstes (in Nachfolge des verstorbenen Hermann Diener) einigermaßen vertraut. Ferner habe ich einige versprengt in partibus erhaltene Dispense aus dem ganzen Spätmittelalter gesammelt (vgl. Anm. 3).

2 Bisher unüberholt Walter von Hofmann, Forschungen zur Geschichte der kurialen Behörden vom Schisma bis zur Reformation, 2 Bde. (Bibl. des kgl. Preußischen Hist. Inst. in Rom 12/13, Rom 1914, ND Turin 1971).

3 Nur wenige regionale Urkundensammlungen haben in partibus erhaltene Pönitentiarie-Urkunden aufgenommen. Zu diesen Ausnahmen gehört Alois Lang (Hrsg.), Acta Salzburgo-Aquilegensia. Quellen zur Geschichte der ehemaligen Kirchenprovinzen Salzburg und Aquileja. Bd. 1: Die Urkunden über die Beziehungen der päpstlichen Kurie zur Provinz und Diözese Salzburg (mit Gurk, Chiemsee, Seckau und Lavant) in der avignonesischen Zeit, 2 Bde. (Quellen und Forsch. zur österr. KiG, Graz 1903/06). Ich habe in meiner eigenen Regestensammlung von Papsturkunden insgesamt 58 Stücke in Regesten ediert: Regesten der in Niedersachsen und Bremen überlieferten Papsturkunden 1198-1503. Bearb. v. Brigide Schwarz Veröffentlichungen der Hist. Komm. für Niedersachsen und Bremen 37, Quellen und Untersuchungen zur Gesch. Niedersachsens im Mittelalter 15, Hannover 1993), vgl. Sachindex s.v.

4 Thomas Frenz, Die Kanzlei der Päpste der Hochrenaissance (1471-1527) (Bibl. des Deutschen Hist. Inst. in Rom 63, Tübingen 1986); ders., Papsturkunden des Mittelalters und der Neuzeit (Hist. Grundwiss. in Einzeldarstellungen 2, Stuttgart 1986); im folgenden zitiert: Frenz, Papsturkunden.

5 Johann Baptist Sägmüller, Lehrbuch des katholischen Kirchenrechts (Freiburg i.Br. 1904) 172f.; im folgenden zitiert: Sajgmüller, Lehrbuch. 
larkleriker ${ }^{6}$ bzw. künftige Regularkleriker (auch weibliche), für die ausschließlich die Pönitentiarie zuständig war. Nur selten beantragten Laien an der Kurie eine Dispens mit dem Ziel, das Erbe ${ }^{7}$ ihrer Eltern anzutreten; das fiel in die Zuständigkeit der Kanzlei.

Eine für höhere Weihen und größeren Pfründenbesitz notwendige apostolische Dispens konnte man sich, wenn man Gelegenheit dazu hatte, auch in partibus beschaffen, nämlich (2) bei päpstlichen Legaten oder Nuntien; deren Dispensations-Kompetenzen, in „Fakultäten" für die jeweilige Legation ${ }^{8}$ speziell festgelegt, waren generell geringer als die der kurialen Instanzen und waren auf bestimmte Kontingente beschränkt ${ }^{9}$ (z.B. bis zu 25 Dispensen für Regularkleriker) ${ }^{10}$. Konkurrierend zum Papst dispensierte übrigens in "meinem" Pontifikat über längere Perioden hinweg noch (3) das Konzil von Basel."

6 In den normativen Quellen für die Kanzlei (dazu unten) wird die Kanzlei offenbar nicht als zuständig für Dispense angesehen, mit denen Eintritt ins Kloster oder Antritt von Klosterämtern ermöglicht werden sollte - und meiner Erinnerung nach auch nicht in den Registern.

7 Siehe Kanzleiregeln Gregors XI. 89, S. 44 und Eugens IV. 50, S. 245 bzw. Nikolaus' V. 52, S. 262, Regulae cancellariae apostolicae. Die päpstlichen Kanzleiregeln von Johannes XXII. bis Nikolaus V. (künftig: RCA). Hrsg. v. Emil von Ottenthal (Innsbruck 1888, ND Aalen 1968). Diese Dispens wird nur mit der Klausel gewährt, daß dadurch denjenigen Erben, die $a b$ intestato erben würden, kein Nachteil entstehen dürfe. Aus meiner Datei zum defectus natalium (dazu Anm. 62), kann ich nur einen Beleg anführen: Johanna mul. Constant. dioc.: de disp. sup. def. nat. (nob.s./nob.s.) ut in hereditatibus succedere val. 22. iun. 46 (S 412 58r). In gewisser Weise hier einschlägig und besonders hübsch der Antrag eines Regensburger Scholaren, dessen Eltern Priester und soluta waren: de lic. accip. totam hereditatem parentum suorum sine alia prole defunctorum (der Bescheid: concessum et sine preiudicio), s.v. Conradus Dragsel, 17. aug. 37 (S 338 $117 \mathrm{r})$.

8 Literatur zum Legationswesen jüngst zusammengestellt bei Erich Meuthen, Die deutsche Legationsreise des Nikolaus von Kues 1451/1452, in: Lebenslehren und Weltentwürfe im Übergang vom Mittelalter zur Neuzeit ... Hrsg. v. Hartmut Boockmann, Bernd Moeller (Abh. der Akad. der Wiss. in Göttingen, Philos.hist. Klasse, 3. Folge, Nr. 179, Göttingen 1989) 421-499, hier 422f. Anm. 7; im folgenden zitiert: Meu-

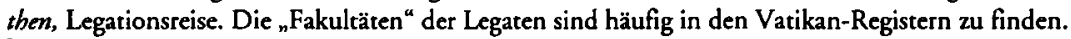

9 Wie sie gehandhabt wurden, wissen wir weniger gut, denn von den Legaten geführte Register ihrer Legationsreise haben sich selten erhalten (z.B. ein Bruchstück des Registers des Legaten Juan Carvajal, Repertorium Germanicum 6, 1, S. XL und Nr. 2678; solche aus dem Pontifikat Eugens IV. sind mir für das Gebiet des Deutschen Reichs nicht bekannt geworden) und von ihnen ausgestellte Dispense sind nur selten gesammelt worden. Eine erste solche Sammlung mit gewissem Anspruch auf Vollständigkeit wird Band I/3 der Acta Cusana, Quellen zur Lebensgeschichte des Nikolaus von Kues, sein, in dem die Dokumente der großen Legationsreise des Nikolaus von Kues gesammelt werden, vgl. Meuthen, Legationsreise (wie Anm. 8). Eine weitere Quelle zur Praxis der Dispensation von Legaten sind narrationes in Suppliken von Petenten, die eine umfänglichere (uberior) Dispens wünschen, als die ihnen von Legaten erteilten.

10 Die Gnadenschätze sollten den Legaten helfen, die Reise zu finanzieren und ihnen ein Maximum an öffentlicher Aufmerksamkeit zu sichern; sie dienten ferner dazu, den Legaten als Vertreter des Papstes gegenüber den ordinarii in angemessener Weise herauszustellen, vgl. Meuthen, Legationsreise (wie Anm. 8), 433-437.

11 Die dispensierende Tätigkeit des Konzils von Basel ist bislang kaum beachtet. Das liegt auch daran, daß die wenigen erhaltenen Register und die Urkunden des Konzils bislang nicht ediert sind; die Edition der Rotaregister und der zwei Suppliken-Register des Konzils von Basel und der 8 "Lateran"-Register aus dem Pontifikat Felix' V. sind im Druck bzw. in Vorbereitung, vgl. meine Ausführungen zum aktuellen Stand der Arbeiten am Repertorium Germanicum: Klerikerkarrieren und Pfründenmarkt, in: QuFiAB 70 (1990) 243-265, hier 265; im folgenden zitiert: Schwarz, Klerikerkarrieren. 
Nun zu Kanzlei und Pönitentiarie ${ }^{12}$ und ihren Kompetenzen, oder - unter anderer Perspektive betrachtet - ihren Angeboten. Die Grundlage dieses Abschnitts sind die einschlägigen normativen Texte, in denen der Papst, bestehenden Kurienbrauch autoritativ interpretierend, mit Geltung prinzipiell nur für seinen Pontifikat, u.a. Teile seiner umfassenden Dispensationsgewalt delegiert; das sind für die Zeit Eugens IV. einmal die - 1438 in einer Konstitution zusammengefaßten - Fakultäten des Großpönitentiars ${ }^{13}$, zum anderen die Kanzleiregeln ${ }^{14}$ mit den darin enthaltenen Fakultäten des Vizekanzlers. Eine generelle normative Abgrenzung der Zuständigkeiten der beiden „Behörden“ gab es nicht.

Der Großpönitentiar hat nach diesen Fakultären folgende Komperenzen:

1) Er kann grundsätzlich alle Unehelichen dispensieren, gleichgültig aus welchen Verbindungen sie hervorgegangen sind. Diese Zuständigkeit gilt jedoch nicht mehr uneingeschränkt, wenn der Petent sich weihen lassen und Kirchenämter bekleiden bzw. Pfründen innehaben will. Außerdem kann der Großpönitentiar nicht dispensieren bei enormitas des Verstoßes der Erzeuger ${ }^{15}$ oder wenn einer von ihnen Prälatenrang hatte ${ }^{16}$, sonst generell zu allen Weihen und - damit zusammenhängend, weil ja niemand ohne Pfründe zu einer

12 Fast alle der in den Supplikenregistern der Pönitentiarie anzutreffenden Sparten von Dispensen und Gnaden wurden auch von der Kanzlei ausgestellt, nicht aber umgekehrt. Der Dispens vom Geburtsmakel ähnlich sind Dispense von Defekten wegen körperlicher Versehrtheit (defectus corporis) und vom fehlenden Weihealter (defectus etatis). Der defectus corporis wurde an der Kurie sehr ernst genommen. Ich habe den Eindruck, daß Eugen IV., der um die rituelle Reinheit des Klerus sehr besorgt war, hier besonders restriktiv entschied. In partibus war man da nicht immer so streng. Der in der Kanzlei überwiegend deklarierte defectus corporis ist Einäugigkeit. Die „Besichtigung“ des Defekts vor einer endgültigen Entscheidung war hochrangigen Kurialen vorbehalten. Sozialhistorisch sehr interessant sind Dispense vom Gebot, die Sprache der Pfarrunterworfenen zu beherrschen (dispensatio super ydeomate, vgl. Anm. 62).

${ }^{13}$ Eugen IV. ließ sie erstmals systematisch zusammenstellen und edierte sie in einer großen "Reformkonstitution" In apostolice dignitatis von 1438-X-14, Ferrara, gedruckt bei Göller, Pönitentiarie, Bd. 1b, 37-47. Die Dispens vom Geburtsmakel ist sehr pauschal in drei Paragraphen abgehandelt (44f.).

14 Die Kanzleiregeln sind von Emil von Ottenthal ediert (wie Anm. 7), bis Nikolaus V. reichend, leider recht unzulänglich; Ergänzungen von Joseph Teige, in: MIOG 17 (1896) 408-440. Ottenthal geht von der irrigen Annahme aus, die Kanzlei habe ihre Kanzlei-Bücher mit jedem Pontifikat auf den aktuellen Stand der geltenden Kanzleiregeln gebracht. Es komme daher nur darauf an, die authentische Fassung des jeweils gültigen Kanzleibuchs zu finden. Die Register, in denen häufig einzelne Kanzleiregeln oder Modifikationen derselben veröffentlicht sind, zog Ottenthal nicht heran. Seine Edition der Kanzleiregeln ist für die einzelnen Pontifikate von sehr unterschiedlicher, z.T. unzureichender Qualität, wie ich bei Untersuchungen über die Regulae cancellariae apostolicae Clemens' VII. und Eugens IV. feststellen konnte. Vgl. auch Andreas Meyer, Spätmittelalterliches Benefizialrecht im Spannungsfeld zwischen päpstlicher Kurie und ordentlicher Kollatur. Forschungsansätze und offene Fragen nebst einem Anhang unpublizierter Kanzleiregeln Nikolaus' V., in: Monumenta iuris canonici, Ser. C: Subsidia 9 (Vatikanstadt 1992) 247-264. Ein weiteres großes Manko ist, daß von von Ottenthal für identisch erklärte Bestimmungen nicht selten beträchtlich verschiedenen Inhalts sind.

15 Dem Gesagten widerspricht scheinbar die Bestimmung 3 der (in Anm. 13 zitierten) Reformkonstitution für die Pönitentiarie (44f), in der unter den Fakultäten des Großpönitentiars auch die qualitercumque et de quocumque etiam damnato coitu nati genannt werden. Hier wird jedoch die Dispensationsgewalt des Großpönitentiars nur auf die Annahme weiterer Benefizien erweitert, für Personen, die bereits eine päpstliche Dispens vorweisen können. Die Dispensation von Abkömmlingen aus solchen Verbindungen blieb Reservatfall, d.h. entweder Angelegenheit der Kanzlei oder Gegenstand besonderer Beauftragung des Großpönitentiars.

${ }_{16}$ RCA (wie Anm. 7) Gregors XI. 55, S. 34 zählt als gravierenden Fall auf die Kombination prelatus/monialis. 
höheren Weihe zugelassen wird - zu allerdings nur einem beneficium simplex, auch einem mit Seelsorgepflichten verbundenen (dann sogenannte prima dispensatio).

2) Diese prima dispensatio kann er in einem weiteren Akt auf ein weiteres einfaches Benefizium ausdehnen, das mit dem ersten kompatibel sein mußte (sogenannte uberior dispensatio), und äußersten Falles noch auf ein drittes, sofern die beiden zusammengenommen nicht ein auskömmliches und für einen Kleriker standesgemäßes Einkommen erbrachten. ${ }^{17}$

Der Vizekanzler (oder sein Stellvertreter in der Kanzlei-Leitung) durfte nach seinen Fakultäten ${ }^{18}$ selbständig folgende standardisierte Fälle erledigen:

1) Die prima dispensatio wie der Großpönitentiar; bei den Hindernissen war jedoch seine Zuständigkeit enger als die des Großpönitentiars; die anderen Fälle mußte der Papst selbst entscheiden.

2) Auch der Vizekanzler konnte die uberior dispensatio gewähren. Hier waren seine Befugnisse größer als die des Großpönitentiars. Er durfte die Annahme von zwei weiteren Benefizien und vor allem von höherrangigen Benefizien bis hin zu Kanonikat und Pfründe und gegebenenfalls einfachem Amt an einer Stiftskirche ${ }^{19}$ erlauben und zwar selbst dann, wenn ein gravierenderes Hindernis vorlag (schließlich hatte in diesem Fall der Papst selbst die prima dispensatio erteilt; dieser konnte hier auch größere Gnaden gewähren). Mit den bisher behandelten (primae und uberiores) dispensationes super defectu natalium quoad beneficia accipienda der Kanzlei und der Pönitentiarie durfte der so Begnadete auf Benefizien seiner Wahl förmlich Anspruch anmelden (akzeptieren), innerhalb des durch die Dispens gezogenen Rahmens. Die Dispens verfiel in dem Augenblick, in dem sie zur Akzeption benutzt worden war, gleichgültig, ob der Dispensierte das Benefizium in Besitz nehmen und behaupten konnte oder nicht; den Anspruch aus der Akzeptation konnte er nicht veräußern.

3) Auf Grund der Zuständigkeit der Kanzlei für Pfründenangelegenheiten durfte der Vizekanzler auch Provisionen mit bestimmten Pfründen (mit bereits vakanten Pfründen ${ }^{20}$ und mit den besonders begehrten reservierten Pfründen, deren Wiederbesetzung dem Papst vorbehalten war) unter gleichzeitiger Gewährung der dafür erforderlichen Dispens erteilen, die hier also Bestandteil einer Pfründenverleihung ist. Eine solche Provision mit Dispens erteilte nur die Kanzlei, und das grundsätzlich nicht gern, wie die Kanzleiregeln zeigen.

17 Göller, Pönitentiarie, Bd. Ib 45 oben.

18 Die Fakultäten des Vizekanzlers finden sich in den RCA (wie Anm. 7) Eugens IV. 97 S. 251f. bzw. RCA (wie Anm. 7) Martins V. 146 S. 224f.; 192 S. 234; 200 S. 235.

19 Dies aber nur nach vorgängiger Genehmigung durch einen Referendar. Zur Entwicklung des päpstlichen Referendariats Ernst Pitz, Supplikensignatur und Briefexpedition an der römischen Kurie im Pontifikat Papst Calixts III. (Bibl. des Deutschen Hist. Inst. in Rom 42, Tübingen 1972) und weiterhin grundlegend Bruno Katterbach, Referendarii utriusque signaturae a Martino V ad Clementem IX et praelati signaturae supplicationum a Martino V ad Leonem XIII (Sussidi per la consultazione dell'Archivio Vaticano II, Studi e testi 55, Vatikanstadt 1931).

20 Wo iure concursus päpstlich Providierte grundsätzlich bevorzugt waren; siehe Andreas Meyer, Zürich und Rom. Ordentliche Kollatur und päpstliche Provisionen am Frau- und Großmünster 1316-1523 (Bibl. des Deutschen Hist. Inst. in Rom 64, Tübingen 1986) 32; im folgenden zitiert: Meyer, Zürich und Rom. 
Auch Dispense, die nicht vom Vizekanzler bewilligt waren, finden sich in den Registern der Kanzlei ${ }^{21}$, denn diejenigen, die der Papst selbst entschied, wurden ja auch in der Kanzlei ausgefertigt. Die Kanzlei ist deshalb die Behörde, die Dispense vom Makel der unehelichen Geburt bei gravierenderen Hindernissen und in komplizierteren Fällen und weitergehende Arten von Dispensen ausstellt, weil ja der Papst allein weder an Fakultäten noch an Regeln gebunden ist und von allen kirchlichen Gesetzen dispensieren kann. ${ }^{22}$

Schließlich konnte allein die Kanzlei einige andere Gnaden für Illegitime anbieten: 1) die besonders begehrte und selten gewährte Dispens vom Geburtsmakel verbunden mit der Erlaubnis, diesen Defekt inskünftig verschweigen zu dürfen, der sogenannten licentia deinceps tacendit ${ }^{23}$. In den Kanzleiregel ${ }^{24}$ wurde immer wieder angeordnet, daß solche Konzessionen künfrig nicht mehr ausgefertigt werden sollten, sogar wenn der Papst (gemäß dem Ideal der liberalitas principis) sie bewilligt hätte ${ }^{25}$, weil sie dem Grundsatz widersprach, daß die Tatsache der Unehelichkeit bei jeder Entscheidung in die Waagschale geworfen werden müsse. Die licentia deinceps tacendi ersparte ihrem Inhaber nicht, den Defekt in den sogenannten Nonobstantien (siehe unten) trotzdem immer wieder deklarieren zu müssen.

2) die Aufhebung von Bestimmungen in Statuten einer Kirche hac vice, die Uneheliche von bestimmten Pfründen ausschließen. - Hier ist nicht gemeint die allgemeine Derogation solcher Statuten als Bestandteil von Dispensen quoad beneficia, die für Pfründen dieser Kategorie ausgestellt wurden, sondern die ausdrückliche Derogation dieser bestimmten Statuten. Die betroffenen Kirchen wehrten sich mit allen Mitteln gegen solche Derogationen, auch unter Heranziehung des Papstes. ${ }^{26}$ Die Durchsetzbarkeit dieser päpstlichen Derogationen wäre nur in partibus aufzuklären. ${ }^{27}$

${ }^{21} \mathrm{Zu}$ den Registerbeständen der "Kanzlei“ im Vatikanischen Archiv (strenggenommen sind im 15. Jahrhundert nur die Lateranregister Kanzleiregister) siehe Leonard E. Boyle, A Survey of the Vatican Archives and of its Medieval Holdings (Toronto 1972); neueste Literatur bei Germano Gualdo, Sussidi per la consultazione dell'Archivio Vaticano. Lo Schedario Garampi, i registri Vaticani, i registri Lateranensi, le „rationes camere“, l'archivio concistoriale (Collectanea Archivi Vaticani 17, Vatikanstadt 1989).

22 Sägmüller, Lehrbuch (wie Anm. 5), 102.

${ }^{23}$ Für die ich eine Datei erstellt habe, vgl. Anm. 62.

24 RCA (wie Anm. 7) Johannes' XXIII. 23, S. 178: die Deklaration des Hindernisses ist inskünftig (!) auch bei Vorliegen einer licentia tacendi nötig, wenn es sich um gratiae beneficiales handelt; Ausnahmen werden gemacht für Inhaber hoher akademischer Grade und für Königs- und Fürstensöhne sowie für Nepoten. Die RCA (wie Anm. 7) Martins V. 87, S. 205 verlangt ebenfalls generell die Deklarierung und verbietet der Kanzlei die Ausstellung solcher Dispense deinceps tacendi in Zukunft - an welche Kanzleiregel sich der Papst selbst natürlich nicht zu halten brauchte.

${ }^{25}$ Noch nicht ausgelieferte sollten kassiert werden. Sollten dennoch solche ausgefertigt werden, seien sie ungültig (RCA [wie Anm. 7] Martins V. 87).

26 RCA (wie Anm. 7) Martins V. 22 S. 191f. betreffend gratiae expectativae; RCA (wie Anm. 7) Eugens IV. 11 S. 241: die Derogation muß ausdrücklich erfolgen, allgemeine Derogierungsklauseln nützen in diesem Fall nichts. Systematischer dann die Regelung in RCA (wie Anm. 7) Nikolaus' V. 79 S. 265.

27 Die Gewährung von Dispens vom Verbot, an derselben Kirche oder gar im selben Benefizium rätig zu sein wie der Erzeuger ( $u$ bi pater - der natürlich nicht notwendig ein illegitimer Erzeuger sein muß), ist nach den RCA (wie Anm. 7) Martins V. 36 S. 195 der Kanzlei verboten. Der Druck der Nachfrage auf den Papst, in diesem Punkt entgegenzukommen, spiegelt sich in dem Verbot an die Kanzlei, solche Dispense auszustellen, auch wenn er sie ausdrücklich genehmigt haben sollte. Hier stehen sich wiederum die liberalitas principis und die Reformauflagen des Konstanzer Konzils einander ausschließend entgegen. Unter 
Vor allem aber kann die Kanzlei, im Unterschied zur Pönitentiarie, bei Dispensationen quoad beneficia bestimmten Kategorien von illegitim geborenen Petenten Vorzugsbedingungen einräumen, nach denselben Kriterien, gemäß denen sie auch sonst Gnadenerweise differenziert zuteilt: mehr und bessere Benefizien (in bezug auf Ansehen und Einnahmen) ${ }^{28}$ und zusätzliche Prärogativen ${ }^{29} \mathrm{zu}$ ihrem Erwerb und Besitz ${ }^{30}$. Solche Bevorzugung gewährte sie bei Würdigkeit des Bewerbers (adelige Abkunft, Graduierung) Verdiensten ${ }^{32}$ und auch bei Protektion. Diese Vorzugsbehandlung erteilte die Kanzlei nicht willkürlich, sondern regelhaft, nach den Traditionen der Kanzlei, die nur teilweise in den Kanzleiregeln schriftlich fixiert sind. Dieser Kanzleibrauch ist eine wichtige normative Quelle, die eigentlich viel zu wenig Beachtung findet. Er ist unter anderem zu fassen in den Bittschriften ${ }^{33}$, die keine Aussicht auf Genehmigung gehabt hätten, wenn sie sich nicht nach dem Kanzleibrauch gerichtet hätten, wenngleich die Petenten ihn natürlich extensiv für sich interpretierten.

Der Papst konnte sich selbstverständlich auch über die Kanzleitradition hinwegsetzen die geschriebenen und ungeschriebenen Kanzleiregeln. Doch sollte dies, wenn es denn geschah, in vollem Bewußtsein aller Implikationen dieser Abweichung von den Normen geschehen. Entsprechend war die Expedition der Kanzlei eingerichtet: Die Referendare, die dem Papst bzw. dem Vizekanzler die Gesuche vortrugen ${ }^{34}$, unterrichteten sie über die hergebrachte Entscheidungspraxis und berieten sie hinsichtlich der Formulierung des Bescheides unter den Bittschriften. ${ }^{35}$ (In diesen Bescheiden kann man auch die persönliche Note jedes einzelnen Papstes studieren, über Kanzleibrauch und aktuelle Nürzlichkeitserwägungen hinaus, die auch eine Rolle spielten.)

Eugen IV. kommen solche Dispense, wenn auch selten, vor, z.B. s.v. Johannes de Echte 2. nov. 37 (S 341 224vs). In der Regel kann man das Verbot durch kreuzweisen Tausch und Rücktausch von Benefizien umgehen. Zur liberalitas principis siehe Hans Kloft, Liberalitas principis. Herkunft und Bedeutung. Studien zur Prinzipatsideologie (Kölner Hist. Abh. 18, Köln/Wien 1970) 183ff.

28 Brigide Schwarz, Römische Kurie und Pfründenmarkt im Spätmittelalter, in: ZHF 20 (1993) 129-152, hier 135; im folgenden zitiert: Schwarz, Pfründenmarkt.

29 Solche Prärogativen waren etwa die licentia permutandi, die licentia tacendi und derogatio statutorum.

30 RCA (wie Anm. 7) Johannes' XXIII. 22 S. 177f.: Eine Provision mit einer Prinzipaldignität für einen Unehelichen muß ausdrücklich diese Tatsache aufführen und der so Begnadete muß adeliger Abkunft oder Graduierter sein.

31 RCA (wie Anm. 7) Gregors XI. 55 S. 34 verfügt generell Vorzugsbedingungen für Studierte.

32 Als solche gelten Fürstendienst und Dienst bei kurialen Großen, insbesondere natürlich beim Papst, siehe Schwarz, Klerikerkarrieren (wie Anm. 11), 252 und dies, Pfründenmarkt (wie Anm. 28), 135.

33 Der Kanzleibrauch ist auch teils aus Formelbüchern, teils aus den unten zu behandelnden reformationes zu rekonstruieren.

34 Dieser entschied dann a), ob das Gesuch rechrens war und b), ob es genügte, die Supplik dem Vizekanzler vorzulegen, oder ob der Papst selbst sie abzeichnen mußte.

35 Kanzleiregeln über die Interpretation von Genehmigungsklauseln unter Suppliken, die nicht nur Dispense enthalten, siehe RCA (wie Anm. 7) Johannes' XXII. 12 S. 4: Grundsätzlich gilt, daß die DispensBitte eigens genehmigt werden muß, vgl. RCA (wie Anm. 7) Gregors XI. 2 S. 26 und Benedikts XIII. 18 S. 127. Später wurde die Kanzlei-Praxis laxer und schränkte diese Bedingung auf "bessere Benefizien“ und weitere Vergünstigungen (etwa Tauschgenehmigung) ein (RCA [wie Anm. 7] Gregors XI. 58 S. 35; RCA [wie Anm. 7] Bonifaz' IX. 14 S. 59). 
In mehreren Prüfungsverfahren kontrollierten dann die domini cancellarie - die Versammlung der oberen Chargen der Kanzlei ${ }^{36}$ - den Wortlaut der Supplik, des Bescheides und des Entwurfs der Bulle, bevor die Dispens die Kanzlei verließ. Sie waren verpflichtet, die päpstlichen Bescheide restrikriv auszulegen, besonders bei den Provisionen mit Dispens. Bei deren Genehmigung war ein fiat ut petitur nur eine Genehmigung der Provision, nicht auch der zugehörigen Dispens-Bitte ${ }^{37}$, die zusätzlich ausdrücklich und in besonderer Weise genehmigt werden mußte, etwa mit et dispensamus ${ }^{38}$, et dispensamus de speciali oder noch komplizierter formuliert. Wie penibel hier die Kanzlei war, wissen wir aus vielen Bitten um Abänderung (reformatio) eines päpstlichen Bescheides in den Registern: der Petent erklärt, daß die domini cancellariae ihm die Ausfertigung der Dispensation in der gewünschten Form verweigerten, weil gegen den Kanzleibrauch verstoßend, und diese nur genehmigt werden könne, wenn der Papst einen anders formulierten Bescheid bewillige ${ }^{39}$, den der Petent vorschlägt. Manchmal folgt der Papst dann dem Vorschlag, manchmal schreibt er aber auch ungerührt den alten Bescheid erneut darunter, womit die Petition zwar formal genehmigt - und deshalb registriert - ist, die Bitte des Petenten aber in der Sache abgelehnt. ${ }^{40}$

Zum Abschluß dieses Abschnitts nenne ich zwei Grundsätze der Kanzleitradition, die man kennen muß, um die Situation und die Aktionen der Illegitimen auf dem Pfründenmarkt ${ }^{41}$ zu verstehen:

1) Nach jeder Transaktion mit Pfründenbesitz verfällt die sie ermöglichende Dispens, also auch die Dispens vom Geburtsmakel.

2) Bei jeder Transaktion müssen der gesamte Pfründenbesitz, inklusive Ansprüchen, und die Dispense deklariert werden in den sogenannten Nonobstantien. ${ }^{42}$ Hier kann schon ein

${ }^{36}$ Der Korrektor, der Auditor litterarum contradictarum und die Abbreviatoren de parco maiori, zuzüglich beigezogene Sachverständige. Sie hielten unter der Leitung des Vizekanzlers in wechselnder Besetzung je nach Materien "Kanzlei“ ab, vgl. Brigide Schwarz, Der "corrector litterarum apostolicarum". Entwicklung des Korrektorenamtes der päpstlichen Kanzlei von Innozenz. III. bis Martin V., in: QuFiAB 54 (1974) 122191 , hier 186.

37 Gelegentlich wird diese Bestimmung abgemildert, daß die Dispens doch, nämlich bei einfachen Benefizien, als mitgemeint präsumiert wird (RCA [wie Anm. 7], Bonifaz' IX. 14 S. 59).

${ }_{38}$ RCA (wie Anm. 7) Johannes' XXII. 12 S. 4, Johannes' XXIII. 11 S. 174 etc. Sogar wenn fiat ut petitur de omnibus beschieden ist, gilt das nicht für die Dispensbitte (RCA [wie Anm. 7] Martins V. 31 S. 193). Die RCA (wie Anm. 7) Gregors XI. 55 S. 34 widerspricht dem nicht, da es hier um einen Ausnahmefall geht.

${ }_{39}$ Der Petent begründet dann seinen neuerlichen Antrag damit, daß er auf Grund des päpstlichen Bescheides nur eine Dispens nach festem Formular (sogenannte dispensatio in forma) erhalten könne, mit der er jedoch, angesichts der scharfen Konkurrenz, nichts anfangen könne.

40 Wir finden daher in den Supplikenregistern in solchen Fällen entweder eine Bitte um eine weitergehende Genehmigung, oder der Petent verzichrete darauf, die Sache weiter zu verfolgen. Zu den Bescheiden unter den Suppliken und den Reaktionen der Petenten darauf siehe auch Sabine Weiss, Kurie, Konzil und Kirchenreform. Salzburg und seine Eigenbistümer im Bannkreis von Papalismus und Konziliarismus (Typoskript Habilschr. Innsbruck 1987). Ich danke Frau Weiss, daß sie mir die Einsichtnahme gewährt hat.

41 Zum Terminus siehe Schwarz, Pfründenmarkt (wie Anm. 28), Anm. 43.

42 RCA (wie Anm. 7) Benedikts XIII. 170 S. 157f.: In allen päpstlichen Gnaden, wie den zahlireichen nove provisiones, provisiones si neutri, gratiae expectativae mußte der defectus natalium deklariert werden, auch wenn das fragliche Benefizium bereits auf Grund einer einschlägigen Dispens erworben wurde; andernfalls galt die Gnade als erschlichen. 
Formfehler (etwa die unrichtige Angabe der unehelichen Eltern) die ganze Gnade hinfällig machen.

Nach diesen Informationen über die Kompetenzen bzw. das Angebot von Kanzlei und Pönitentiarie nun einige Beobachtungen über die Praxis bzw. die Nachfrage, die sich ergeben ${ }^{43}$ aus langjähriger Beschäftigung mit den Kanzlei-Registern und dem Studium der von L. Schmugge und seinen Mitarbeitern untersuchten Supplikenregister-Bände der Pönitentiarie aus der Zeit Pius' II. (an der Rechtslage hatte sich seit Eugen IV. nichts Grundsätzliches verändert $\left.{ }^{44}\right)$. Wie schon deutlich wurde, waren für bestimmte Kategorien von Dispensen super defectu natialium Kanzlei und Pönitentiarie zuständig, so daß die Interessenten insoweit die Wahl hatten. Wofür man sich entschied, wenn man wählen konnte, war sicher nicht vom Zufall bestimmt, sondern von Überlegungen darüber, wo ein günstigeres Verhältnis von Aufwand und Ertrag zu erreichen war.

Aufwendig war es auf jeden Fall, sich um eine apostolische Dispens zu bemühen; bei Abwesenheit des Petenten von der Kurie ${ }^{45}$ schon wegen der Notwendigkeit, einen Agenten, "Prokurator ${ }^{466}$, heranzuziehen. ${ }^{47}$ Der Prokurator ${ }^{48}$ mußte zunächst herausfinden, ob sein Klient in der Kanzlei oder in der Pönitentiarie ${ }^{49}$ besser bedient war - sofern beides möglich war. Dazu mußte er scharf rechnen, denn die Dispense der Kanzlei waren teurer, insbesondere natürlich die, die der Papst selbst genehmigte; sowohl die reinen Bearbeitungsgebühren waren in der Kanzlei wesentlich höher ${ }^{50}$ als auch die sogenannten

43 Diese Beobachtungen sind nicht auf exakte Auszählungen im RG 5 gestützt. Dazu ist das Manuskript noch nicht weit genug gediehen.

44 Bde. 7-10. Grundlegend war weiterhin die Reformkonstitution Eugens IV. von 1438, vgl. Göller. Pönitentiarie, Bd. 2a $13 \mathrm{f}$.

45 Diese Notwendigkeit erübrigte sich, wenn ein für die Zwecke des Petenten mit günstigen Fakultäten ausgestatteter Legat in erreichbarer Nähe war. Die persönliche Anwesenheit des Petenten an der Kurie ersparte einige Kosten und ermöglichte Transaktionen, die sonst mit Kosten und Zeitverlust verbunden waren (vgl. Anm. 53).

46 Zur Geschichte der Prokuratoren und ihrer Ausdifferenzierung in verschiedene Klassen im Spätmitrelalter siehe Patrick N. R. Zutshi, Proctors Acting for English Petitioners in the Chancery of the Avignon Popes, in: JEcclH 35 (1984) 15-29; Christiane Schuchard, Die Deutschen an der päpstlichen Kurie im späten Mittelalter (1378-1447) (Bibl. des Deutschen Hist. Inst. in Rom 65, Tübingen 1987) 67-70; im folgenden zitiert: Schuchard, Deutsche; Brigide Schwarz, Die römische Kurie im Zeitalter des Schismas und der Reformkonzilien, in: Institutionen und Geschichte. Theoretische Aspekte und mittelalterliche Befunde. Hrsg, v. Gert Melville (Norm und Struktur. Studien zum sozialen Wandel in Mittelalter und früher Neuzeit 1, Köln/Weimar/Wien 1992) 231-258.

47 Wegen dieser Einschaltung eines Agenten, der die Daten seines Auftraggebers nicht immer genau notiert hatte, kommen nicht selten Fehler in den Supplikenregistern vor (etwa bei der Angabe des Standes der Eltern), die dann öfter Gegenstand von Bitten um Nachbesserung sind.

48 Sogenannte reformationes, die nicht selten wohl auch vorgeschoben sind, um doch noch die gewünschte Form der Bulle zu erreichen, unter günstigeren Umständen, siehe Schwarz, Pfründenmarkt (wie Anm. 28), 137.

49 Bei einer ganzen Reihe von Stücken ist nicht erkennbar, warum der Petent sich hier an die Kanzlei und nicht an die Pönitentiarie wandte. Übrigens war das auch schon den Zeitgenossen nicht klar, denn es gibt Gesuche, wegen einer verschollenen Dispens sowohl in den Kanzleiregistern wie in denen der Pönitentiarie nachzusehen, vgl. das Fallbeispiel unten.

50 Brigide Schwarz, Die Organisation kurialer Schreiberkollegien von ihrer Enstehung bis zur Mitte des 15. Jahrhunderts (Bibl. des Deutschen Hist. Inst. in Rom 37, Tübingen 1972) 25-32, 261f. und die dort genannte Literatur. 
Kompositionen, Zahlungen, die an den Papst zu entrichten waren bei Dispensationen wie auch bei anderen Gewährungen einer besonderen Gunst. ${ }^{51}$ Nicht umsonst zu haben waren auch der in komplizierten Fällen eventuell benötigte fachkundige Rat für den Prokurator und die Fürsprache von Protektoren, die man bei der Kanzlei einschalten konnte. Günstig gestellt war hier, wer Beziehungen zur Kurie hatte, oder mittelalterlich gesprochen: wer Kuriale kannte oder einen, der Kuriale kannte. ${ }^{52}$

Ob die Einschaltung der Kanzlei möglich oder vorteilhafter war, richtete sich nach den besonderen Umständen des Falles. Nach meinen Beobachtungen wurde beim Großpönitentiar vor allem um primae dispensationes nachgefragt zum Eintritt in den Klerus sowie zur Annahme niederer Weihen, was ich im Kanzleimaterial nur selten gefunden habe ${ }^{53}$ (wobei der Vorbehalt zu machen ist, daß einschlägige Unterlagen verlorengegangen sein könnten; dazu gleich). Interessenten für eine uberior dispensatio des Großpönitentiars waren offenbar Leute, die bescheidener waren - oder sein mußten. ${ }^{54}$ Insgesamt waren solche Gesuche dort nicht sehr zahlreich.

Fragt man, welche Personen, welche Fälle eher in den Registern der Kanzlei zu finden sind, so scheint mir: schwererwiegende Verstöße und kompliziertere Fälle, zumal wenn sie Rechtsstreit nach sich zogen; Petenten höheren Standes (allerdings auch solche servilis conditionis). Bei den Ausfertigungen fällt auf, daß hier die gewährten Gnaden freier formuliert und daß bei der Bestellung der Exekutoren häufiger und weiter von der kanonischen Norm abgewichen wird. Diese Beobachtungen finde ich dadurch bestätigt, daß sich

51 Wolfgang Reinhard u.a., Ämterhandel in Rom zwischen 1534 und 1621, in: Ämterhandel im Spätmirtelalter und im 16. Jahrhundert (Einzelveröffentlichungen der Hist. Komm. zu Berlin 45, Berlin 1984) 43.

52 Daher kann man bei denen, die sehr viel um päpstliche Gnaden einkommen, intensive Beziehungen zu Kurialen annehmen (Schuchard, Deutsche [wie Anm. 46], 185ff.). Diese können, wenn auch nicht immer auf direktem Wege, fast immer nachgewiesen werden (Brigide Schwarz, Patronage und Klientel in der spätmittelalterlichen Kirche, in: QuFiAB 68 [1988] 284-310, und dies., Pfründenmarkr [wie Anm. 28], $145 f$.

53 Es wäre interessant zu erfahren, ob sich an der Kurie Anwesende eher an die Pönitentiarie oder die Kanzlei wandten. Leider geben das die Gesuche der Pönitentiarie nie, die der Kanzlei nicht durchgängig an. Zuweilen ist es jedoch zu erschließen, vgl. Sabine Weiss, Salzburger am Hof Martins V. in Rom (14201431), in: RQA 86 (1991) 53-77, hier 62ff. An der Kurie Anwesende konnten den Pfründprozeß gleich in der Kanzlei anmelden, was für sie von großem Vorteil war. Ende des 15. Jahrhunderts wurde offenbar das Verfahren vereinfacht, indem von der Pönitentiarie Dispensierte über die Pönitentiarie ihren Pfründprozeß anmelden konnten, was ihnen Umstände ersparte, vgl. die bei Göller, Pönitentiarie, Bd. 2b 142f. abgedruckte Taxordnung von ca. 1480-1500, S. 142, Abschnitt 1-2: Statt der $121 / 2$ Groschen, wie für einen Abwesenden üblich, kostere für einen Anwesenden die einfache Dispens cum processu 18 1/2 Groschen propter processum; aus demselben Grund ist auch der Preis der uberior dispensatio für Anwesende höher (ebd., Abschnitt 9).

54 Den uberiores dispensationes der Pönitentiarie-Supplikenregister ist leider nichts zu entnehmen über das aliud beneficium, das sie so erwerben. Unklar bleibt deshalb, ob die Petenten erwa zusätzlich die Genehmigung erhielten, eins der beiden Benefizien zu tauschen. In der Pönitentiarie wie in der Kanzlei ist stets nur angegeben, ob der Petent aufgrund der vorgängigen prima dispensatio (die er genau deklarieren muß) bereits ein Benefizium besitzt oder nicht. Das wirkt sich auch auf die Kosten aus, vgl. die Taxordnung der Pönitentiarie von ca. 1480-1500 (vorige Anm.). 
Ähnliches feststellen ließ über die in der Kanzlei sehr viel seltener vorkommenden Dispense vom defectus corporis, vom defectus etatis und die dispensationes matrimoniales."

Die Entscheidung für Kanzlei oder Pönitentiarie richtete sich offenbar auch nach den Chancen auf dem Pfründenmarkt. Es scheint, daß sich um die teuerere und schwerer zu erhaltende, auf die Pfründenwünsche genau zugeschneiderte Dispens vom Geburtsmakel der Kanzlei eher derjenige bewarb, der schon gute Aussichten auf „bessere" Pfründen besaß. Die übrigen versuchten eher, mittels ihrer allgemeinen Dispense quoad beneficia Hand auf ein Benefiz zu legen, sobald sich ihnen die Chance dazu bot, um auf dem hart umkämpften Pfründenmarkt mitzuhalten. Doch gerieten sie da leicht in Konflikt mit den Canones: wenn etwa ein Petent ${ }^{56}$ auf Grund einer Dispens ein Benefizium akzeptiert hatte ${ }^{57}$, dieses dann aber ohne Einholung einer neuen Dispens gegen ein anderes getauscht hatte, war damit sein gesamter Pfründenbesitz eo ipso verwirkt und er war inhabilis geworden; denn jede Pfründen-Transaktion bedurfte ja - wie gesagt - prinzipiell einer neuen Dispens (sofern man nicht von vorneherein in der Kanzlei eine weitergehende Dispens erhalten hatte) ${ }^{58}$. Wenn das aufkam, half nur noch eine dispensatio super inhabilitate, die voraussetzte, daß der Anspruch auf den verwirkten Pfründenbesitz mittels resignatio in die Hände des Dispensierenden (Papst oder Vizekanzler) aufgegeben wurde, der sie dann mittels nova provisio dem Reuigen - nach Zahlung einer saftigen compositio wieder verlieh zuzüglich der erforderlichen uberior dispensatio ${ }^{59}$. Die Risiken der rehabilitatio waren, daß man beim Wiedererwerb der verwirkten Pfründen der offenen Konkurrenz ausgesetzt war.

Manche Uneheliche brauchten sich offenbar wenig um ihre Dispens-Basis zu scheren und behaupteten sich trotzdem in den Pfründen, wie zu ersehen ist aus Bitten um Pfründen, die frei seien, weil ihr Besitzer keine ausreichende Dispens habe. Eine interessante, aber aus dem Registermaterial nicht zu beantwortende Frage wäre: Hatte jemand, der

55 Für die ich Dateien angelegt habe (vgl. Anm. 62).

56 Ein Beispiel: Am 16. Januar 1438 erwirkte ein Bittsteller in der Kanzlei eine Dispens wegen Irregularität ex delicto, in der Terminologie des neueren Kirchenrechts (Sägmüller, Lehrbuch [wie Anm. 5]), bzw. cine rehabilitatio im Jargon der Kanzlei. Die Inhabilität hatte er sich zugezogen, weil er eine (prima) Dispens vom Geburtsmakel des Großpönitentiars „überzogen" hatte: Er hatte nämlich als Scholar kraft dieser Dispens eine Pfarrkirche akzeptiert, mit der er natürlich nichts anfangen konnte, wenn er nicht binnen eines Jahres zum Priester geweiht war - was unmöglich war. Diese Dispens hätte er auch in der Kanzlei bekommen können und zwar relativ einfach, weil der Vizekanzler solche selbständig erteilen konnte. Er hatte die Pfarrkirche dann gegen ein Objekt getauscht, das er behalten konnte (eine Kapelle, mit einem schönen Einkommen von 4 bzw. 6 Mark). Da er jedoch keine Genehmigung zu dem Tausch hatte, die die Pönitentiarie damals auch gar nicht ausstellen konnte, hatte er gegen die Kanones verstoßen, war inhabilis, hatte eo ipso alles Recht sowohl auf die Pfarrkirche wie auf die Kapelle verloren. Ihm blieb nur, sich an die Kanzlei zu wenden wegen Rehabilitation, was nur möglich war nach Resignation aller Ansprüche auf die genannten Pfründen in die Hände des Vizekanzlers. Sofern er aus den zu Unrechr besessenen Pfründen Einkommen bezogen hatte (fructus male percepti), hatte er eine saftige Buße (compositio) zu zahlen. Danach erteilte die Kanzlei eine erneute Provision mit der Kapelle und gewährte die dazu nötige uberior dispensatio, s.v. Burchardus de Ingenheim (lugenheim) (ASV S 343 220rs, L 357 203rs).

57 Vielleicht auch vigore uberioris dispensationis ein weiteres hinzugewonnen hatte.

58 Das scheint später möglich gewesen zu sein, vgl. Taxordnung von ca. 1480-1500, Göller, Pönitentiarie, Bd. 2b 142, Abschnitt 4: pro legitimatione ad duo beneficia compatibilia unacum permutatione eorundem [a] quocumque genitlo] gr. $161 / 2$.

59 Er riskierte damit, daß ein anderer sich ebenfalls mir den Pfründen providieren ließ. 
etwa von seinem unehelichen adeligen Erzeuger (kraft Patronatsrecht) mit Pfründen gut versorgt war, eine uberior dispensatio wirklich nötig, wenn es keine Konkurrenten gab und eine Anzeige beim Papst ${ }^{60}$, der den Anzeigenden im Erfolgsfall mit den verfallenen Pfründen providierte, sinnlos schien?

Wie wir sahen, hatten die Inhaber von Dispensen quoad beneficia accipienda höchst unterschiedliche Chancen, auf dem Markt auftauchende Pfründen zu akzeptieren (Illegitimer ist eben nicht gleich Illegitimem). Ähnlich differenziert behandelte die Kanzlei auch andere Kleriker, die ein Benefizium akzeptieren wollten mit Hilfe von päpstlichen Anwartschaften auf eine bzw. mehrere inskünftig freiwerdende Pfründen (gratiae expectativae). Diese reichten von der Kommungratie nach festem Formular für arme Kleriker bis hin zur Spezialgratie für besonders Bevorzugte. In der Regel war unter Eugen IV. die gratia expectativa auf bestimmte Pfründen einer Kirche bzw. einer Kollatur beschränkt, dafür enthielt sie aber stets auch die päpstliche Provision, die preiswerter war als die direkte päpstliche Provision mit einer vakanten oder mit einer reservierten Pfründe. Um die jeweils benötigte Provision mußre sich der Illegitime noch zusätzlich zu seiner Dispens bemühen, je nach Vakanzgrund beim Papst oder beim Ordinarius. Wie die Dispensatio quoad beneficia verfiel auch die gratia expectativa nach Inanspruchnahme eines Titels. Diese Ähnlichkeiten waren es wohl, die die Kanzlei veranlaßten, die beiden Gnaden nach recht ähnlichem Formular ${ }^{6 l}$ zu behandeln. Wenn man bedenkt, daß die gratia expectativa für die spätmittelalterlichen Kleriker der wichtigste Zugang zum Pfründenmarkt war (wie Andreas Meyer nachgewiesen hat), wird man eine ähnlich große Bedeutung der dispensatio quoad beneficia für Illegitime vermuten dürfen.

Es geht hier nicht um eine sozialgeschichtliche Analyse des Materials über Illegitime in den Kanzlei-Registern. Aber ich möchte zum Schluß noch ein paar Bemerkungen über die Auswertung der Register ${ }^{62}$ für solche Analysen machen. Es liegen keine speziellen Register

60 Mir sind Beauftragungen, ein Verfahren einzuleiten mit dem Ziel, den gegen die Canones Verstoßenden seiner Benefizien zu entheben, sogenannte commissiones privationis, mit dieser Begründung nicht erinnerlich, was aber bei dem riesigen Material nicht viel besagt. Öber die sozialgeschichtliche Relevanz der commissiones privationis, die der Konkurrent um die Pfründe zu betreiben hatte, siehe Schwarz, Klerikerkarrieren (wie Anm. 11), 257.

${ }_{61}$ Bei den überaus zahlreichen nove provisiones etwa wird der Besitzanspruch in derselben Weise ausgedrückt: - qui vigore gratie expectative - bzw. qui vigore dispensationis super defectu natalium - accepit beneficium NN. Zwei bei der Herstellung des Repertorium Germanicum vielgebrauchte Textbausteine, die auch die Zusammenführung der beiden Sachverhalte in Indices erlauben.

62 Um die Aussagen dieser Studie mit Material zur Dispensationspraxis der Kanzlei belegen zu können, habe ich einige Dateien zusammengestellt, und zwar aus unterschiedlich großen Ausschnitten aus dem Rohmanuskript des RG-Bandes 5: Die Datei zum (1) defectus natalium ist allein aus den Viten Johannes Aa bis Johannes $M$ genommen, was mir ausreichend schien (Johannes ist bei weitem der im Repertorium Germanicum am häufigsten vorkommende Name; er scheint ständisch indifferent vergeben worden zu sein). Einige Dateien zu anderen Dispensen stammen aus dem gesamten Material von Aa bis Johannes M: (2) Dispense wegen Mangels körperlicher Tüchtigkeit (defectus corporis), (3) Dispense vom Gebot, die Sprache der Pfarrunterworfenen zu beherrschen (super ydeomate), (4) Dispense von inkurrierter Inhabilität und Irregularität. „Umfassend" sind ferner die Dateien über die Gesuche um (5) Erlaubnis, Dispense verschweigen zu dürfen (licentia oder dispensatio deinceps tacendi), (6) zur Geschichte der Pönitentiarie und (7) zu den Vollmachten und der Tätigkeit der Legaten und Nuntien. Die Datei zu den (8) Dispensen von Ehehindernissen hingegen wurde aus einem kleineren Ausschnitt des Manuskripts genommen, weil sie sonst zu umfangreich geworden wäre. Keine Datei habe ich über Inkompatibilitäts-Dispense erstellt, die nur im 
nur für Dispense vor. Ich halte es aber für nicht unwahrscheinlich, daß es Kurz-Register von Kommundispensen gegeben hat, die nicht aufbewahrt worden sind, wie andere Serien auch, die nicht fiskalisch erheblich waren (so die Expektativenregister in communi forma pauperum, von denen durch Zufall zwei Exemplare erhalten sind ${ }^{63}$, und die Register der "gemeinen" littere de gratia und littere de iusticia, die gänzlich verloren sind $\left.{ }^{64}\right)$. Auf jeden Fall sind viele Bände der einschlägigen, erhaltenen Registerserien verlorengegangen ${ }^{65}$, was quantifizierende Aussagen erschwert.

Es sind mehrere Register-Serien, in denen dispensationes super defectu natalium und Material darüber zu finden sind. Zu beachten ist, daß man für jeden einzelnen Fall Register verschiedener Serien heranziehen muß, weil der Vorgang dort in verschiedenen Expeditionsstufen festgehalten wurde. Die Supplikenregister enthalten die nach Angaben der Petenten formulierten, sozialgeschichtlich besonders interessanten Suppliken, die Bescheide der Päpste darauf und etwaige Nachbesserungen. Die Ausfertigungen von Dispensen finden sich in den Auslaufregistern (den Lateran- und - selten - den Vatikanregistern), die allein die Nonobstantien stets vollständig enthalten ${ }^{66}$, die Kommissare (bei den Dispensen) und die für die Rekonstruktion des Networks so wichtigen Exekutoren (bei Provisionen mit Dispensen). Zu beachten ist, daß es nur zu einem Bruchteil der Bittschriften ${ }^{67}$ Ausfertigungen gab. Angaben über Zahlungen, die mit der Erteilung von Dispensen verbunden sind, finden sich in den Kameralregistern (genauer gesagt in den sonstigen Kameralregistern, denn, strenggenommen, zählen dazu auch die Suppliken- und

Zusammenhang mit dem Pfründenmarkt-Geschehen verständlich sind, auch keine über Dispense vom Mangel des gesetzlichen Alters (defectus etatis), für die ich mich mit einer stichprobenweisen Auszählung begnügr habe. - Diese Dateien leiden unter manchen Schwächen, die mit dem Bearbeitungsstand des Repertorium Germanicum-Bandes zusammenhängen. - Für alle auf EDV-Träger aufgenommenen Bände des Repertorium Germanicum (die jedoch z.T. erheblich weniger Daten enthalten als RG 5, vgl. unten Anm. 77) können für derartige Fragen nun sogenannte KWIC-Indices hergestellt werden, Indices, die einen Suchbegriff mit den ihn umgebenden Wörtern präsentieren.

${ }^{63}$ Andreas Meyer, Arme Kleriker auf Pfründensuche. Eine Studie über das ,in forma pauperum“-Register Gregors XII. von 1407 und über päpstliche Anwartschaften im Spätmitrelalter (Forsch. zur kirchlichen Rechtsgesch. und zum Kirchenrecht 20, Köln 1990); Andreas Sohn, „Pauperes clerici“ an der römischen Kurie Pauls II. (1464-1471). Ein Beitrag zur prosopographischen Auswertung eines Vatikanregisters (Reg. Vat. 541), in: Vinculum Sociecatis. Fschr. für J. Wollasch. Hrsg. v. Franz Neiske u.a. (Sigmaringendorf 1991) 276-301.

64 Vgl. Peter Herde, Die "Registra contradictarum“ des Vatikanischen Archivs 1575-1799, in: Paleographica, diplomatica et archivistica. Studi in onore di Giulio Batelli 2 (Rom 1979) 409-444; Frenz, Papsturkunden (wie Anm. 4), 74 nimmt keinerlei Registrierung, auch nicht in Kladden, an. Diese Akten hatten alle keine fiskalische Erheblichkeit, wie übrigens auch der größte Teil der politischen Korrespondenz.

65 Über die Verluste in den Beständen der drei großen Registerserien der "Kanzlei“ vgl. Hermann Diener, Die großen Registerserien im Vatikanischen Archiv (1378-1523). Hinweise und Hilfsmittel zu ihrer Benurzung und Auswertung, in: QuFiAB 51(1971) 305-368 (auch Separatdruck), hier 307, 343. Was die Verteilung der Materien über die Pontifikatsjahre angeht, ist stets zu bedenken, daß sehr viele Einträge ins erste Jahr datiert sind, die - für uns im einzelnen nicht verifizierbar - in spätere Jahre gehören, vgl. das Fallbeispiel unten.

66 In den Supplikenregistern ist zunehmend die Gnade anzutreffen, daß der Petent seine Nonobstantien erst bei Ausstellung der Bulle in der Kanzlei deklarieren muß, ein erheblicher Gewinn für den Prokurator, der inzwischen präzisere Information einholen kann.

67 Verhältnis etwa 1:10, errechnet von Meyer, Zürich und Rom (wie Anm. 20), 52, für den Pontifikat Martins V. 
die Vatikanregister). Schließlich sind Angaben über Dispense zu finden in allen Stücken, die Nonobstantien ${ }^{68}$ haben.

Eine große Schwierigkeit liegt darin, daß Material über Dispense in den Registern nicht leicht zu finden ist. ${ }^{69}$ Zwar haben die Kanzlei-Register Eugens IV. eine grobe Unterteilung der Einträge nach Materien (hier einschlägig etwa de diversis formis) und nach genehmigender Instanz (Papst oder Vizekanzler, was in den Kanzlei-Registern stets klar zu erkennen ist an der Form des Bescheides unter den Gesuchen, per fiat oder per concessum $)^{70}$, doch eigene Lagen für den Betreff Dispens vom Geburtsmakel wie in der Pönitentiarie gibt es in keinem Kanzlei-Register. Für die Interpretation der Registereinträge verweise ich nochmals auf das schon dargestellte Problem: Die Formeln fiat ut petitur oder fiat in forma heißen nicht einfach, daß alles Erbetene gewährt wird. Zu beachten ist auch, daß durch die Einschaltung von Prokuratoren nicht selten Fehler in den Registern vorkommen (etwa bei der Angabe des Standes der Eltern), die des öfteren Gegenstand von Bitten um Nachbesserungen ${ }^{71}$ sind, und demjenigen entgehen, der die entsprechende Nachbesserung nicht beachtet.

Aber der Forscher braucht nicht vorzeitig zu verzagen angesichts aller dieser Schwierigkeiten: Es gibt ja das Repertorium Germanicum, das ihm hier weiterhilft, wenn auch leider nicht so weit, wie es ihm wünschenswert wäre. Mißlich ist zunächst, daß die Bände 1-4 (Schisma bis Martin V. inklusive) gar keine Sachindices haben, die Bände 6-7 relativ grob sortierte; erst ab Band 8 (1993 erschienen) wird es tiefer sortierte Indices geben ${ }^{72}$, so auch für den Band über Eugen IV. Weitere Einschränkungen der Nutzungsmöglichkeiten ergeben sich aus bestimmten Eigentümlichkeiten des Repertorium Germanicum: a) Beschränkung auf deutsche Belange, was aber weit ausgelegt wird ${ }^{73}$; b) Beschränkung der Aufnahme von Informationen durch den prosopographischen Zweck des Unternehmens $^{74}$; dadurch etwa Nicht-Berücksichtigung kanonistischer Fragestellungen; c) die

68 Alle pfründenrelevanten Stücke. Im Pontifikat Eugens IV. noch die meisten Suppliken (vgl. Anm. 66) und alle Ausfertigungen; die Nonobstantien meist in der Form: non obstante defectu natalium (folgt Angaben zu Vater und Mutter) super quo cum ipso NN dispensatum est.

69 Die Zugehörigkeit der einzelnen Stücke zu bestimmten, sachlich ausgerichteten Lagen konnte ich für diese Studie nicht mehr rekonstruieren, nachdem ich schon vorher die zig-tausende Scheden der Regesten des Repertorium Germanicum $V$ aus ihrem Archivzusammenhang genommen und zu den üblichen (mehr als 10000 ) Repertorium-Germanicum-Viten zusammensortiert hatte. Das wird erst wieder möglich sein, wenn die Fundstellenindices erstellt sein werden. Solche Indices haben nun alle späteren Bände des RG und des RPG, der neuen Reihe mit den Pönitentiar-Betreffen.

70 Etwas abweichend die Praxis bei Eugen IV., der viel krank war: statt mit fiat sind von ihm genehmigte Suppliken meist mit concessum in presentia pape unterzeichnet.

${ }^{71}$ Eine in unserem Zusammenhang hübsche Nachbesserung (perinde valere): Die Angabe des Erzeugers "lediger Ritter" in einer uberior dispensatio (qui vig. uberioris disp. sup. def. nat. [mil.s./.s.] ad 3 benef. c. lic. perm. par. eccl. de Ebren Herbip. dio. assec. est) sei unrichtig, weil pater eius temp. nativitatis eius tantum militaris war ; der Petent schließt sicherheitshalber die Bitte um nova provisio mit der genannten Pfarrkirche (6 m. a.) an, s.v. Johannes de Helb 20. sept. 36 (ASV S 326 277v).

72 Von Karl Borchardt zusammengestellt.

73 Über den Gebrauch „Deutsches Reich“ im Repertorium Germanicum siehe Dieter Brosius, Das Repertorium Germanicum, in: Das Deutsche Historische Institut in Rom 1888-1988 (Bibl. des Deutschen Hist. Inst. in Rom 70, Tübingen 1990) 123-166.

74 Ebd. 
weitere Verknappung der Informationen in den einzelnen Viten gemäß den Richtlinien. Das bedeutet z.B., daß die ungemein häufigen Nennungen des defectus natalium (def. nat. im Repertorium Germanicum) in den oben genannten Nonobstantien, die bei der Aufnahme aus den Registern natürlich vermerkt wurden, nach der ersten Nennung weggelassen werden, es sei denn, die Angaben differierten. Wo der interessierte Forscher mit den Angaben im Repertorium Germanicum nicht auskommt, müßte er sich an die Karteien im Deurschen Historischen Institut in Rom begeben oder natürlich ad fontes im Vatikanischen Archiv. 


\section{Fallbeispiel}

Im Frühjahr 1433 geht in der Kanzlei das Gesuch eines Albertus de Rischach um uberior dispensatio vom Geburtsmakel ein. ${ }^{75}$ Die für dieses Gesuch notwendige erste päpstliche Dispens kann nicht vorgelegt werden. Der Prokurator des (offenbar fernen) Mandanten hat als Anhaltspunkt nur das Datum. Er läßt die Dispens - was teuer war - in den Registern suchen, und zwar erst in den Auslauf-Registern der Kanzlei, den Lateranregistern ${ }^{76}$, wo man sie anscheinend am ehesten erwartete, dann in denen des Großpönitentiars und zuletzt in den Supplikenregistern, wo das Suchen wegen der großen Datenmassen und Unübersichtlichkeit sehr erschwert war. Nach vergeblicher Suche (auf deren mögliche Ursachen ich oben eingegangen bin) scheint man sich in der Kanzlei mit einer entsprechenden „eidesstattlichen Erklärung" begnügt zu haben, was man nicht so ohne weiteres tat.

Der Petent ist Rektor einer Pfarrkirche; sein Geburts-Defekt: Vater: verheirateter Ritter/Mutter: ledig. Mittels der erweiterten Dispensation will er zusätzlich zu einer Pfarrkirche, die er besizzt und die gut ausgestatter ist (18 Mark Silber!), noch 4 (!) andere Benefizien akzeptieren. Eines der ins Auge gefaßten Benefizien ist eine Domherrenstelle in Konstanz; das verrät die von der Kanzlei gewährte Derogation der Statuten der Domkirche. Die Dispens ist nur in den Lateranregistern erhalten und zeigt, daß der Petent weitere Prärogativen, außer der genannten Derogation der Statuten, erwirkt hat: eine Vordatierung auf das Krönungsdatum des Papstes 11. März 1431 (zu erschließen aus dem Expeditionsdatum). Die Dispens, die üblicherweise in Form einer commissio 77 an den Ordinarius ausgestellt wird, erfolgt in unserem Fall an den Propst von Bischofszell statt an den Bischof von Konstanz ${ }^{78}$; ob das ebenfalls Bestandreil der Vorzugsbehandlung ist, kann ich mit „Bordmitteln" nicht klären.

75 RG 5: Albertus de Rischach rect. par. eccl. s. Martini in Iveringen Constant. dioc. (de iure patron. Laic.), c. quo s.d. 16 febr. 29 disp. est sup. def. nat. (mil c. et s.), (que disp. autem nec in apostolicarum nec maior. penitentiarie litt. aut supplic. registratis invenitur): disp. sup. d. def. ad prep. eccl. s. Pelagii Episcopaliscelle Constant. dioc. et ut unac. d. par. eccl. $(18 \mathrm{~m}$. a.) 4 alia benef. obtin. valeat; $n$. o. statutis eccl. maior. Constant. 11 . mart. 31 (exped. 9. mai 33) (ASV L 309 56vss).

76 Das ist mit registra apostolicarum litterarum (vgl. Anm. 75) gemeint.

7 Leider werden in den anderen Bänden des Repertorium Germanicum die so wichtigen Kommissionen bzw. Exekutoren nicht aufgenommen.

78 Es gab um diese Zeit Wirren um das Bistum, die auch mit Rücktritt des gegenwärtigen Bischofs Otto III. von Hachberg am 15. September 1433 nicht beendet waren, vgl. dazu REC, Bd. 3 Nr. 9530, 9585, 9590ff. und $U$. Janson, Otto von Hachberg (1388-1451), Bischof von Konstanz und sein Traktat

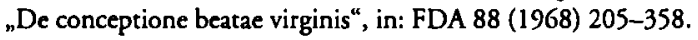


